\title{
The choice of Methods of Dynamic Errors Compensation in Motion Control of Elastic Mechatronic Systems
}

\author{
N. K. Kuznetsov ${ }^{1, *}, I . A . \operatorname{Iov}^{2}, A . A . \operatorname{Iov}^{2}$, and E. S. Dolgih ${ }^{1}$ \\ ${ }^{1}$ Irkutsk National Research Technical University, 83 Lermontov St., Irkutsk, 664074, Russian \\ Federation \\ ${ }^{2} \ll$ SLC-JH» Ltd., Mira St., 101 bld, Moscow, 129085, Russian Federation
}

\begin{abstract}
In this paper, using the example of a two-mass calculation scheme, which can be used to describe the dynamic properties of many mechatronic systems with elastic elements, we present the results of research on finding control actions that provide compensation of dynamic errors based on solving the inverse dynamic problems according to a given law of change of the force in an elastic element. It is shown that the found control actions in the form of time dependences make it possible not to impose any restrictions on the methods of their technical implementation and thereby provide a comprehensive solution the problem of compensating for dynamic errors caused by elastic deformations of mechanical elements.
\end{abstract}

\section{Introduction}

An increase in the speeds and loads of modern technological and transport machines (metalworking machines, machining centers and flexible production modules, industrial and manipulation robots, ground and underground transport and technological complexes, walking and bucket wheel excavators, etc.), which can be attributed to the class of mechatronic systems, due to the presence of digital control systems, makes it necessary to take into account the elastic properties of the design and develop methods and means of compensating the dynamic errors caused by oscillatory movements of the actuators, which disrupt the accuracy of functioning, increase the time of performing operations, reduce the strength of the main elements, efficiency, and reliability works [1-6].

At present time the problem of compensation the dynamic errors of mechatronic systems is solved by the methods of machine mechanics associated with the choice of design parameters (inertial, stiffness and dissipative) actuators [7-11], and by the methods of control theory using the purposeful choice of the structure and parameters of the controllers of the electric drive control system [12-19]. The technical solutions proposed in these works are based more on engineering experience and intuition than on a scientific and methodological basis, which would allow formalizing the procedure for the structuralparametric synthesis of controls means the dynamic state of mechatronic systems.

\footnotetext{
*Corresponding author: knik@istu.edu,
} 
In this regard, it becomes necessary to develop a unified approach to the problems of controlling the dynamic state of such systems, based on a rational combination of methods of machine mechanics with the potential capabilities of control systems and modern means of information processing and decision making. An effective tool for the implementation of such an approach can be the use of the concept of inverse dynamic problems, which makes it possible to form the control of the dynamic state of mechatronic systems based on the required nature of controlled motion [20]. As shown in [21, 22], the control actions obtained in the form of time dependences based on solving the inverse dynamic problems for a given form of the transient process allows not to impose any restrictions on the methods and means of their technical implementation and thereby provide a comprehensive approach to the problem of compensating dynamic errors caused by elastic deformations of mechanical elements.

In this paper, using the example of a two-mass calculation scheme, which can describe the dynamic properties of many mechatronic systems with elastic element, we present the results of studies on determining the control actions that compensate the dynamic errors based on solving the inverse dynamic problems according to a given law of change of the force in an elastic element.

\section{Object and method of investigation}

As an object of research, we choose a two-mass calculation scheme shown in fig. 1, on which the following designations are adopted: Electric Converter - control signal converter to the drive torque $M_{D V} ; J_{1}$ is the inertia of the shaft motor and reduced moment of inertia the transmission mechanism; $J_{2}$ is the equivalent moment of inertia of the actuator; $\varphi_{1}$ and $\varphi_{2}$ are generalized coordinates of reduced masses; $c_{12}$ and $b_{12}$ are reduced coefficients of stiffness and viscous friction of the elastic element; $M_{C}$ is the load torque [21].

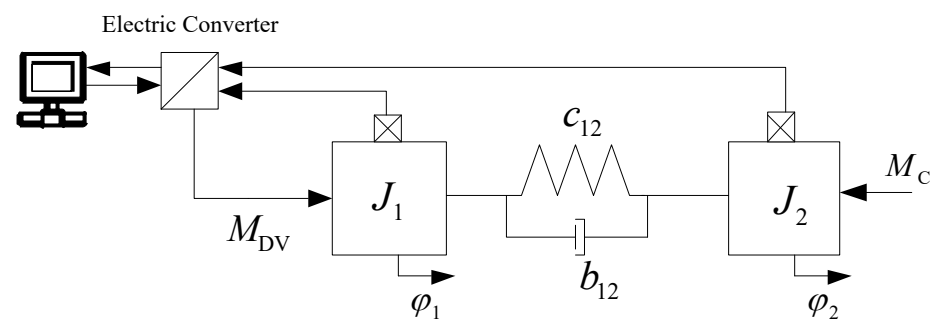

Fig. 1. Calculation scheme of mechatronic system

The linearized differential equations describing the behavior of the mechanical part of the system, without taking into account the dynamic properties of the electric drive and the control signal converter, can be represented as follows:

$$
\begin{aligned}
& J_{1} \dot{\varphi}_{1} s=M_{\mathrm{DV}}-\frac{c_{12} \Delta \dot{\varphi}_{12}}{s}-b_{12} \Delta \dot{\varphi}_{12}, \\
& J_{2} \dot{\varphi}_{2} s=\frac{c_{12} \Delta \dot{\varphi}_{12}}{s}+b_{12} \Delta \dot{\varphi}_{12}-M_{C} .
\end{aligned}
$$

Where $\Delta \dot{\varphi}_{12}=\dot{\varphi}_{1}-\dot{\varphi}_{2}$ is the velocities of the elastic deformation; $s=\frac{d}{d t}$ is a Laplace operator. 
It is known that the main source of dynamic errors in a mechatronic system is an elastic element, in which kinetic energy is continuously converted into potential energy of elastic deformations. To synthesize the control action based on the solution of the inverse dynamic problem we set the required law of change of the force in the elastic element based on the specified parameters of the mechanical part, taking into account the possibility of its physical feasibility [20].

For this purpose, we solve the equations of motion (1) and (2) with respect to the force in the elastic element $M_{12}=\frac{c_{12}+b_{12} s}{s} \Delta \dot{\varphi}_{12}$, provided that $M_{C}=0$

$$
M_{12} s^{2}+b_{12} J_{12} M_{12} s+\omega_{12}^{2} M_{12}=\frac{c_{12}+b_{12} s}{J_{1}} M_{D V},
$$

where $\omega_{12}^{2}=\frac{c_{12}\left(J_{1}+J_{2}\right)}{J_{1} J_{2}}$ is the natural frequency oscillation of a two-mass mechanical system; $J_{12}=\frac{J_{1}+J_{2}}{J_{1} J_{2}}$ is the ratio of the inertia.

The required law of change of the force in the elastic element can be obtained based on the choice of the roots of the left side of equation (3), as was done, for example, in [23] based on the given damping coefficient $\xi$. Neglecting the coefficient $b_{12}$ on the right side of equation (3), this law will have the form

$$
M_{12}=e^{\alpha t}(\cos \beta t+\sin \beta t)
$$

where $\alpha \pm j \beta$ are the roots of the equation of the characteristic polynomial.

When setting (4), the type of the transient process will be determined by the relationship between the parameters $\alpha$ and $\beta$, and correspond to the motion of the system (3) under a unit step function.

We find the required law of change of the force in the elastic element taking into account the right side of equation (3) and a unit step function $M_{D V}=1, t$ at zero initial conditions. Inhomogeneous equation (3) at $M_{D V}=1, t$ can be represented as equivalent to the homogeneous with nonzero initial conditions $M_{12}(0)=J_{1} /\left(J_{1}+J_{2}\right)$ and $\dot{M}_{12}(0)=b_{12} / J_{1}$ [24]. Solution this equation is written as

$$
M_{12}=e^{\alpha t}\left(C_{1} \cos \beta t+C_{2} \sin \beta t\right)
$$

in which constants $C_{1}$ and $C_{2}$ are defined by expressions

$$
C_{1}=J_{2} /\left(J_{1}+J_{2}\right), C_{2}=-\frac{1}{\beta}\left(\frac{J_{2} \alpha}{J_{1}+J_{2}}+\frac{b_{12}}{J_{1}}\right) \text {. }
$$

Considering that $\alpha=-\frac{b_{12} J_{12}}{2}$ [25], the solution equation (3) can be represented in the following form

$$
M_{12}=\frac{J_{2}}{J_{1}+J_{2}} e^{\alpha t}\left(\cos \beta t+\frac{\alpha}{\beta} \sin \beta t\right) .
$$

Comparing expressions (4) and (5), it should be noted that in addition to the new constant $J_{2} /\left(J_{1}+J_{2}\right)$, which determines the steady motion and does not affect its transient process, a new factor appears at the sinus function $\alpha / \beta$. With the coefficient of relative damping equal to $\xi=0,707(|\alpha|=|\beta|)$, the law of change of the force in the elastic element, taking into account the right side in equation (3), can be written as 


$$
M_{12}=e^{\alpha t}(\cos \beta t-\sin \beta t) .
$$

In expressions (4) and (6), the parameters $\alpha$ and $\beta$ can be determined based on the standard forms of the characteristic polynomial [24,25].

We find the control actions that implement the given laws of change of the forces in the elastic element (4) and (6) in the mechatronic system (3) by solving the inverse dynamic problem the algorithm of which is described in detail in [21]. The calculation results are presented in the form of a table 1 in which the left column corresponds to the law (4), the right one to the law (6). It should be noted that (4) and (6) define the law of change of the force in the elastic element in the form of time dependencies, which makes it possible not to impose restrictions on the method of technical implementation of the obtained control actions. The resulting dependencies can be implemented in the form of feedbacks on the force in the elastic element and its derivative.

Table 1. The calculation results of control actions

\begin{tabular}{|c|c|}
\hline \multicolumn{2}{|c|}{ Derivatives up to second orders. } \\
\hline$\left.M_{12} S=(\alpha+\beta) e^{\alpha t} \cos \beta t+(\alpha-\beta) e^{\alpha t} \sin \beta t\right)$ & $\left.M_{12}^{\prime} s=(\alpha+\beta) e^{\alpha t} \cos \beta t+(\alpha-\beta) e^{\alpha t} \sin \beta t\right)$ \\
\hline $\begin{array}{l}M_{12} s^{2}=\left(\alpha^{2}+2 \alpha \beta-\beta^{2}\right) e^{\alpha t} \cos \beta t+ \\
+\left(\alpha^{2}-2 \alpha \beta-\beta^{2}\right) e^{\alpha t} \sin \beta t\end{array}$ & $\begin{array}{l}M_{12}^{\prime} s^{2}=\left(\alpha^{2}+2 \alpha \beta-\beta^{2}\right) e^{\alpha t} \cos \beta t+ \\
+\left(\alpha^{2}-2 \alpha \beta-\beta^{2}\right) e^{\alpha t} \sin \beta t\end{array}$ \\
\hline \multicolumn{2}{|c|}{ Parameters of equation (3) after substitution of derivatives } \\
\hline $\begin{array}{l}M_{D V}\left(c_{12}+b_{12} p\right)= \\
J_{1} K_{1} e^{\alpha t} \cos \beta t+J_{1} K_{2} e^{\alpha t} \sin \beta t\end{array}$ & $\begin{array}{l}M_{D V}\left(c_{12}+b_{12} p\right)= \\
J_{1} K_{1}^{\prime} e^{\alpha t} \cos \beta t+J_{1} K_{2}^{\prime} e^{\alpha t} \sin \beta t\end{array}$ \\
\hline $\begin{array}{l}K_{1}=\alpha^{2}+2 \alpha \beta-\beta^{2}+b_{12} J_{12}(\alpha+\beta)+\omega_{12}^{2} \\
K_{2}=\alpha^{2}-2 \alpha \beta-\beta^{2}+b_{12} J_{12}(\alpha-\beta)+\omega_{12}^{2}\end{array}$ & $\begin{array}{l}K_{1}^{\prime}=\alpha^{2}-2 \alpha \beta-\beta^{2}+b_{12} J_{12}(\alpha-\beta)+\omega_{12}^{2} \\
K_{2}^{\prime}=-\alpha^{2}-2 \alpha \beta+\beta^{2}-b_{12} J_{12}(\alpha+\beta)-\omega_{12}^{2}\end{array}$ \\
\hline \multicolumn{2}{|c|}{ Laws of change of control actions } \\
\hline$M_{D V}=B_{1} e^{\alpha t} \cos \beta t+B_{2} e^{\alpha t} \sin \beta t$ & $M_{D V}=B_{1}^{\prime} e^{\alpha t} \cos \beta t+B_{2}^{\prime} e^{\alpha t} \sin \beta t$ \\
\hline $\begin{array}{l}B_{1}=\frac{J_{1}\left[K_{1}\left(c_{12}+b_{12} \alpha\right)-b_{12} \beta K_{2}\right]}{\left(c_{12}+b_{12} \alpha\right)^{2}+b_{12}^{2} \beta^{2}} \\
B_{2}=\frac{J_{1}\left[K_{2}\left(c_{12}+b_{12} \alpha\right)+b_{12} \beta K_{1}\right]}{\left(c_{12}+b_{12} \alpha\right)^{2}+b_{12}^{2} \beta^{2}}\end{array}$ & $\begin{aligned} B_{1}^{\prime} & =\frac{J_{1}\left[K_{1}^{\prime}\left(c_{12}+b_{12} \alpha\right)-b_{12} \beta K_{2}^{\prime}\right]}{\left(c_{12}+b_{12} \alpha\right)^{2}+b_{12}^{2} \beta^{2}} \\
B_{2}^{\prime} & =\frac{J_{1}\left[K_{2}^{\prime}\left(c_{12}+b_{12} \alpha\right)+b_{12} \beta K_{1}^{\prime}\right]}{\left(c_{12}+b_{12} \alpha\right)^{2}+b_{12}^{2} \beta^{2}}\end{aligned}$ \\
\hline \multicolumn{2}{|c|}{ Control actions based on feedbacks } \\
\hline$M_{D V}=\left(K_{V} s+K_{A}\right) M_{12}$ & $M_{D V}=\left(K_{V}^{\prime} s+K_{A}^{\prime}\right) M_{12}$ \\
\hline$K_{V}=\frac{B_{1}-B_{2}}{2 \beta}$ & $K_{V}^{\prime}=-\frac{B_{1}^{\prime}+B_{2}^{\prime}}{2 \beta}$ \\
\hline$K_{A}=\frac{B_{1}(\beta-\alpha)+B_{2}(\alpha+\beta)}{2 \beta}$ & $K_{A}^{\prime}=\frac{B_{1}^{\prime}(\alpha+\beta)+B_{2}^{\prime}(\alpha-\beta)}{2 \beta}$ \\
\hline
\end{tabular}

Compare the obtained feedback coefficients $K_{V}$ and $K_{V}^{\prime}, K_{A}$ and $K_{A}^{\prime}$. Equating the coefficients $K_{V}=K_{V}^{\prime}$, we obtain the following ratio

$$
\frac{B_{1}-B_{2}}{2 \beta}=-\frac{B_{1}^{\prime}+B_{2}^{\prime}}{2 \beta} \text {. }
$$


Replacing $B_{1}$ and $B_{2}, B_{1}^{\prime}$ and $B_{2}^{\prime}$ their values from the table 1 and denoting the left side of the ratio by the expression

$$
A_{1}=\left(c_{12}+b_{12} \alpha\right)\left(4 \alpha \beta+2 b_{12} J_{12} \beta\right)-2 b_{12} \beta\left(\alpha^{2}-\beta^{2}+b_{12} J_{12} \alpha+\omega_{12}^{2}\right),
$$

after some transformations, we get the same value on the right side of the relation.

The fulfillment of the equality condition indicates that the coefficients $K_{V}$ and $K_{V}^{\prime}$ for two different laws of change of the force in the elastic element are identically equal. Similarly, after equalizing the coefficients $K_{A}=K_{A}^{\prime}$

$$
\frac{B_{1}(\beta-\alpha)+B_{2}(\alpha+\beta)}{2 \beta}=\frac{B_{1}^{\prime}(\alpha+\beta)+B_{2}^{\prime}(\alpha-\beta)}{2 \beta},
$$

we obtain identical expressions for the left and right sides of the last relation, equal to

$$
A_{2}=\left[\left(c_{12}+b_{12} \alpha\right) \beta+b_{12} \alpha \beta\right]\left(\alpha^{2}-\beta^{2}+b_{12} J_{12} \alpha+\omega_{12}^{2}\right)+\left[\left(c_{12}+b_{12} \alpha\right) \alpha-b_{12} \beta^{2}\right)\left(-2 \alpha \beta-b_{12} J_{12} \beta\right) \text {. }
$$

Thus, we can conclude that the law (6) based on the feedback principle leads to the implementation of the motion of the mechatronic system determined by law (4), under a unit step function and the condition that the coefficient $b_{12}$ on the right side of equation (3) is equal to zero. The structural scheme of the synthesized mechatronic system is shown in fig. 2. It should be noted that this statement is valid only for mechatronic systems with weak internal damping when $b_{12}$ is relatively small.

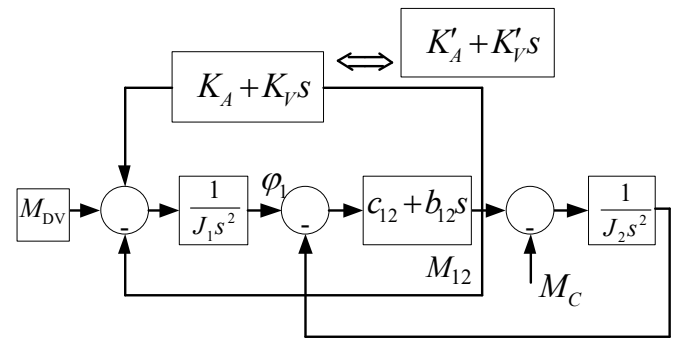

Fig. 2. Structural scheme of a system with feedback control

We estimate the influence of the parameter $b_{12}$ in the right side of the differential equation (3) on the nature of the transient processes when the law of change of the force in the elastic element is implemented in the form (4). Taking the coefficient in the right side of the equation $b_{12}=0$, with relative damping $\xi=0.707$, we obtain the solution of the equation under a unit step function

$$
M_{12}=e^{\alpha t}\left(C_{1} \cos \beta t+C_{2} \sin \beta t\right)=e^{\alpha t} \sqrt{C_{1}^{2}+C_{2}^{2}} \sin (\beta t+\varphi),
$$

where $\operatorname{tg} \varphi=\frac{C_{1}}{C_{2}}, C_{1}=C_{2}=\frac{J_{2}}{J_{1}+J_{2}}$.

Using the left column of the table 1 , we write down the differential equation in which $b_{12}$ from the right is not equal to zero, and the control is implemented based on the feedback principle

$$
\left(1+\frac{K_{V} b_{12}}{J_{1}}\right) M_{12} s^{2}+\left(b_{12} J_{12}+\frac{K_{A} b_{12}}{J_{1}}+\frac{K_{V} c_{12}}{J_{1}}\right) M_{12} s+\left(\omega_{12}^{2}+\frac{K_{A} c_{12}}{J_{1}}\right) M_{12}=\frac{c_{12}+b_{12} s}{J_{1}} M_{D V} .
$$

The solution of this equation with a unit step function can also be written in the form (7), however, the constants $C_{1}^{\prime}$ and $C_{2}^{\prime}$ will differ, since in the characteristic polynomial the 
highest is not equal to one. By calculating $\operatorname{tg} \varphi^{\prime}=\frac{C_{1}^{\prime}}{C_{2}^{\prime}}$ and comparing it with a similar parameter of expression (7), it will be possible to estimate the influence of the right side of equation (3) on the nature of the motion of the system.

As it was established above, the control based on the feedback principle does not allow creating a change of the force in the elastic element in the form (6), obtained taking into account the right side of equation (3). We find the control action in the parameter space of the system using equation (8). Reducing it to a normal form by dividing all its members by the coefficient at the highest derivative and equating the coefficients at the corresponding powers in its left side to the coefficients of equation (3), we obtain the following relations:

$$
\begin{gathered}
\frac{\omega_{12}^{2} J_{1}+K_{A} c_{12}}{J_{1}+K_{V} b_{12}}=\widetilde{\omega}_{12}^{2}, \\
\frac{b_{12} J_{12} J_{1}+K_{A} b_{12}+K_{V} c_{12}}{J_{1}+K_{V} b_{12}}=\widetilde{b}_{12} \widetilde{J}_{12} .
\end{gathered}
$$

Where $\widetilde{\omega}_{12}^{2}$ and $\widetilde{b}_{12} \widetilde{J}_{12}$ are new parameters of the mechatronic system. It should be noted that the expression $\widetilde{\omega}_{12}^{2}$ includes a parameter $\widetilde{J}_{12}$, which will not cause any special difficulties in the calculation $\tilde{b}_{12}$.

\section{Research and Discussion}

We will evaluate the effectiveness of using the proposed approach on a specific example of a mechatronic system, with parameters close to the parameters of a real digging mechanism of a dragline: $\quad J_{1}=572 \mathrm{~kg} \cdot \mathrm{m}^{2}, \quad J_{2}=60 \mathrm{~kg} \cdot \mathrm{m}^{2}, \quad c_{12}=7500 \mathrm{~N} \cdot \mathrm{m} / \mathrm{rad}$, $b_{12}=150 \mathrm{~N} \cdot \mathrm{m} \cdot \mathrm{s} / \mathrm{rad}$. Under these parameters, the calculated values of the roots of the characteristic polynomial, at $\xi=0.707$, were $\alpha=-8.308$ and $\beta=8.311$, and the feedback coefficients turned out to be: $K_{V}=K_{V}^{\prime}=-1.46$, and $K_{A}=K_{A}^{\prime}=-4.037$. The constants in the solutions of differential equations turned out to be equal to the following values: $C_{1}=C_{2}=0.095, C_{1}^{\prime}=0.06869$ and $C_{2}^{\prime}=0.0458$.

Calculating the values of $\operatorname{tg} \varphi=1$ and $\operatorname{tg} \varphi^{\prime}=1.499$, we establish that the presence of the viscous friction coefficient $b_{12}$ in the right side of equation (3) when constructing a control based on the feedback principle will lead to a phase shift upward, and, consequently, to an increase in the rate of the transient process while maintaining the overshoot value. By way of illustration, fig. 3a shows the graphs of changes of the forces in the elastic element $M_{12}$ obtained with a unit step function $M_{D V}=1, t$, in which the number 1 corresponds to the adopted law (4), and 2 corresponds to the law found taking into account the right side in equation (3). 


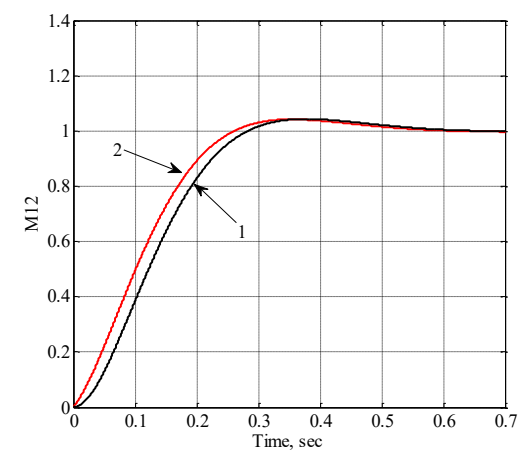

a)

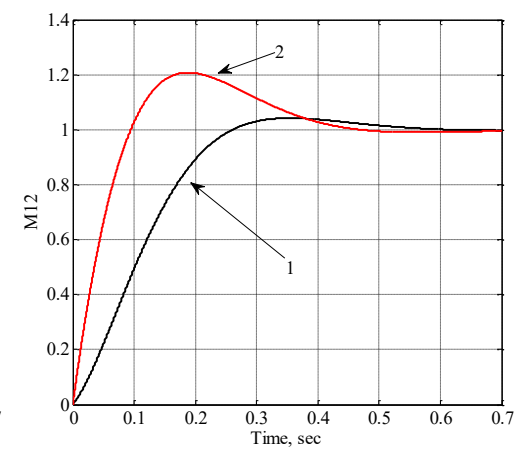

b)

Fig. 3. Graphs of changes of the forces in the elastic element

Substituting the numerical values of the parameters of the mechanism and feedbacks in (9) and (10) we find new values of the parameters of the mechatronic system $\widetilde{\omega}_{12}^{2}$ and $\widetilde{b}_{12} \widetilde{J}_{12}$. Comparing them with the parameters of the original system shows that the natural frequency of oscillations of the system $\widetilde{\omega}_{12}^{2}=\omega_{12}^{2}=138.11 \mathrm{rad} / \mathrm{sec}$ has not changed, and, therefore, retained their values and parameters $c_{12}, J_{1}$ and $J_{2}$. The value $\tilde{b}_{12}$ is determined from relation (10) with known parameters $c_{12}, J_{1}$ and $J_{2}$, coefficients $K_{V}$ and $K_{A}$, as a result of which we obtain $\tilde{b}_{12}=903 \mathrm{~N} \cdot \mathrm{m} \cdot \mathrm{s} / \mathrm{rad}$. Substituting new parameters into the mechatronic system (3), make sure that the changing of the force in the elastic element occurs according to the prescribed law (6), which is confirmed by the graphs of changes of this force in fig. $3 b$, obtained with control based on feedback (curve 1) and by choosing parameters (curve 2 ).

\section{Conclusion}

The studies carried out have confirmed the possibility of using the concept of inverse dynamic problems to develop a unified approach to the problems of controlling the dynamic state of mechatronic systems by a rational combination of the methods of machine mechanics and the potential capabilities of motion control systems. It is shown that the choice of the desired law of change of the force in the elastic element based on the characteristic polynomial, describing the dynamic properties of the mechatronic system, allows the implementation of control actions through feedback, but in this case, it is necessary to take into account the parameters of viscous friction in the right side of the differential equation, which will lead to an increase the speed of transient processes. The formation of the force in the elastic element based on the law obtained taking into account the right side of the original differential equation, is possible by changing the parameters of the original mechatronic system, which can be determined by comparing the original characteristic polynomial and the polynomial obtained when implementing the feedback control. The obtained analytical dependencies reflect the direct connection of the parameters of the mechatronic system with the quality indicators of the transient process and allows to formalize the procedure of structural-parametric synthesis of controlling means the dynamic state of such systems. 


\section{References}

1. Svinin V.M. Milling with modulated cutting speed: monograph (Publ. ISTU, Irkutsk, 207)

2. R.H. Sharipov, The calculation of the loads acting on the construction of the EKG-5 excavator, Mining informational and analytical bulletin (scientific and technical journal), v. 6, pp. 113-116 (2010)

3. Lagunova Yu.A., Komissarov A.P., V.S. Shestakov et al, Mechanical engineering. Mining machines. T IV-24 (Mechanical engineering, Moscow, 2011)

4. A.V. Savilov, A.S. Pyatykh, Vibration effect on accuracy and quality of hole surface under drilling, Vestnik ISTU, v. 12(83), pp. 103-111 (2013)

5. Stepanov A.G., Kornyakov M.V. Machine Dynamics (Publ. ISTU, Irkutsk, 2014)

6. Ilyin S.R., Ilina S.S., Samusya V.I., Mechanics of mine hoist: Monograph (National Mining University, Dnepropetrovsk, 2014)

7. Poderny R.Yu. Mechanical equipment of mine. Book for university (Mining Media Group, Moscow, 2011)

8. A.A. Gubenko, V.P. Svinarchuk, Review and analysis of design parameters of elasticdamping devices, Scientific Annals of MGGU, v. 1, pp. 12-16 (2011)

9. H.N. Sultonov, L.I. Kantovich, Improving the reliability of excavator travel mechanism drive gear - face power shovel, Coal, v. 6, pp. 30-32 (2014)

10. I.L. Pastoev, I L, E.P. Zelenovskii, Active damping dynamic load in traction chains front planing machine, Depon. Manuscript Mining informational and analytical bulletin (scientific an technical journal), v. 1 (1993)

11. N.K. Kuznetsov, I.A. Iov, A.A. Iov, E.S. Dolgih, Development and research of elasticdamping device with variable stiffness, IOP Conference Series: Materials Science and Engineering, 971, 042016, DOI 10.1088/1757-899X/971/4/042016. (2020)

12. Bortsov Yu.A., Sokolovsky G.G., Automated electric drive with elastic connections (Energoatomizdat, St. Petersburg, 1992)

13. Burgin B. Sh. Analysis and synthesis of two-mass electromechanical systems (Novosibirsk Electrotechnical Institute, Novosibirsk, 1992)

14. Pyatibratov G.Ya. Development of the theory and practice of effort control in electromechanical systems with elastic connections, dissertation (South Russian State Technical University, Novocherkassk, 2000)

15. A.V. Lyakhomsky, S.N. Reshetnyak, Methods for studying the correction of coordinates in electric drives of mine hoist, Electrica, 4, pp. 33-37 (2009)

16. Lyakhomskiy A.V., Fashilenko V.N., Control of electromechanical mining machine systems (Moscow State Mining University, Moscow, 2004)

17. V.I. Vasiliev, Ways of reducing dynamic loads in the cable of mine hoist by systems of automatically controlled safety braking, Steel ropes: Collection of articles. scientific. tr. MAISK, Odessa, Astroprint, v. 8, pp. 18-29 (2010)

18. S.V. Solov'ev, D.A. Kuziev, Dependence of dynamics of the working process of a career dragline on elastic-damping parameters of its traction mechanism, Coal, 2, pp. 60-62 (2014)

19. V.V. Alekseev, A.P. Emelyanov, A.E. Kozyaruk, Analysis of the dynamic performance of a variable-frequency induction motor drive using various control structures and algorithms, Russian Electrical Engineering, v. 87(4), pp. 181-188 (2016)

20. Krut'ko P.D. Inverse dynamics problems of control systems: Linear models (Science, Moscow, 1987) 
21. N.K. Kuznetsov, E.S. Dolgih, I.A. Iov, Synthesis of optimal structures of the motion controller of elastic mechatronic systems, International Multi-Conference on Industrial Engineering and Modern Technologies (FarEastCon), Vladivostok, Russia, pp. 1-6, (2019) doi: 10.1109/FarEastCon.2019.8934439

22. N.K. Kuznetsov, I.A. Iov, E.S. Dolgih, Development and Research of Motion Control Algorithms of Elastic Mechatronic Systems, International Multi-Conference on Industrial Engineering and Modern Technologies (FarEastCon), Vladivostok, Russia, pp. 1-5, (2020) doi: 10.1109/FarEastCon50210.2020.9271077

23. N.K. Kuznetsov, I.A. Iov, E.S. Dolgih, Structural-parametric synthesis of the electric drive control system of excavators digging mechanisms based on the inverse dynamic problems concept, Proceed. of the 6th International Conference on Industrial Engineering, 1, pp. 647-656 (2020).

24. Krasovsky A.A., Pospelov G.S., Fundamentals of automation and technical cybernetics (Gosenergoizdat, Moscow, 1962)

25. Dorf R., Bishop R., Modern control systems (Eighth Edition, Addison-Wesley, 2002) 